\title{
Front Matter: Volume 10944
}

, "Front Matter: Volume 10944," Proc. SPIE 10944, Practical Holography XXXIII: Displays, Materials, and Applications, 1094401 (24 May 2019); doi: $10.1117 / 12.2531382$

SPIE. Event: SPIE OPTO, 2019, San Francisco, California, United States 


\title{
PROCEEDINGS OF SPIE
}

\section{Practical Holography XXXIII: Displays, Materials, and Applications}

\author{
Hans I. Bjelkhagen \\ V. Michael Bove Jr. \\ Editors
}

4-5 February 2019

San Francisco, California, United States

Sponsored and Published by

SPIE 
The papers in this volume were part of the technical conference cited on the cover and title page. Papers were selected and subject to review by the editors and conference program committee. Some conference presentations may not be available for publication. Additional papers and presentation recordings may be available online in the SPIE Digital Library at SPIEDigitalLibrary.org.

The papers reflect the work and thoughts of the authors and are published herein as submitted. The publisher is not responsible for the validity of the information or for any outcomes resulting from reliance thereon.

Please use the following format to cite material from these proceedings:

Author(s), "Title of Paper," in Practical Holography XXXIII: Displays, Materials, and Applications, edited by Hans I. Bjelkhagen, V. Michael Bove Jr., Proceedings of SPIE Vol. 10944 (SPIE, Bellingham, WA, 2019) Seven-digit Article CID Number.

ISSN: 0277-786X

ISSN: 1996-756X (electronic)

ISBN: 9781510625303

ISBN: 9781510625310 (electronic)

Published by

SPIE

P.O. Box 10, Bellingham, Washington 98227-0010 USA

Telephone +1 3606763290 (Pacific Time) · Fax +1 3606471445

SPIE.org

Copyright (C) 2019, Society of Photo-Optical Instrumentation Engineers.

Copying of material in this book for internal or personal use, or for the internal or personal use of specific clients, beyond the fair use provisions granted by the U.S. Copyright Law is authorized by SPIE subject to payment of copying fees. The Transactional Reporting Service base fee for this volume is $\$ 18.00$ per article (or portion thereof), which should be paid directly to the Copyright Clearance Center (CCC), 222 Rosewood Drive, Danvers, MA 01923. Payment may also be made electronically through CCC Online at copyright.com. Other copying for republication, resale, advertising or promotion, or any form of systematic or multiple reproduction of any material in this book is prohibited except with permission in writing from the publisher. The CCC fee code is 0277 $786 \mathrm{X} / 19 / \$ 18.00$.

Printed in the United States of America by Curran Associates, Inc., under license from SPIE.

Publication of record for individual papers is online in the SPIE Digital Library.

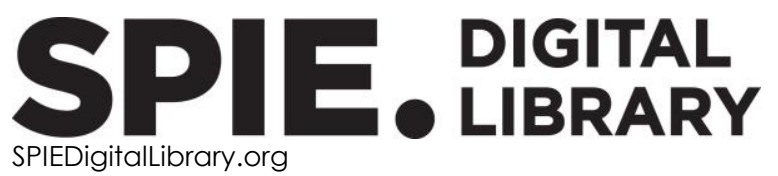

Paper Numbering: Proceedings of SPIE follow an e-First publication model. A unique citation identifier (CID) number is assigned to each article at the time of publication. Utilization of CIDs allows articles to be fully citable as soon as they are published online, and connects the same identifier to all online and print versions of the publication. SPIE uses a seven-digit CID article numbering system structured as follows:

- The first five digits correspond to the SPIE volume number.

- The last two digits indicate publication order within the volume using a Base 36 numbering system employing both numerals and letters. These two-number sets start with $00,01,02,03,04$, 05, 06, 07, 08, 09, OA, OB ... 0Z, followed by 10-1Z, 20-2Z, etc. The CID Number appears on each page of the manuscript. 


\title{
Contents
}

\author{
vii Authors \\ ix Conference Committee
}

\section{SESSION $1 \quad$ MATERIALS AND PROCESSES I}

1094402 Integration of volume holographic optical elements (vHOE) made with Bayfol ${ }^{\circledR}$ HX into plastic optical parts [10944-1]

1094403 Azo-polymers for holographic recording: photo-assisted holography and surface relief gratings [10944-2]

$1094405 \quad$ High efficiency diffractive optical element design and fabrication in a two-stage photopolymer [10944-4]

\section{SESSION 2 MATERIALS AND PROCESSES II}

$1094408 \quad$ Lasers for holographic applications: important performance parameters and relevant laser technologies [10944-7]

1094409 Study of the behavior of diffraction gratings with twin grooves [10944-8]

10944 OA Complex amplitude modulation metasurface with dual resonance in transmission mode [10944-9]

\section{SESSION 3 ART AND PERCEPTION}

10944 OC A comparative analysis of virtual-reality art-making software for the production of VR digital holograms [10944-11]

10944 OD A holographic representation of three-dimensional J9 space [10944-12]

10944 OE Design and implementation of a full-color holographic thaumatrope [10944-13]

\section{SESSION $4 \quad$ APPLICATIONS}

10944 OF Manufacturable transparent holographic components for HUD applications [10944-14] 
10944 OG Compact augmented-reality glasses using holographic optical element combiner [10944-15]

$10944 \mathrm{OH} \quad$ Improving head-up display with waveguides and holographic optical elements [10944-16]

10944 Ol Proper autofocus for better particle measurements [10944-17]

10944 OJ Three-dimensional particle localization with common-path digital holographic microscopy [10944-18]

10944 OL Digital holographic camera for plankton monitoring [10944-20]

\section{SESSION 5 DIGITAL HOLOGRAPHY I}

10944 OM Holographic stereogram printing based on digitally computed content generation platform [10944-21]

10944 ON Multiplexed pixelated hologram recording process for retinal projection device [10944-22]

$1094400 \quad$ Expressible-depth control method in digital holographic display [10944-23]

$109440 Q \quad$ Algorithm for phase-displacement conversion from reflection digital holographic interferometry [10944-25]

10944 OR Effect of point pitch on speckle noise in computer-generated hologram using point-based method [10944-26]

\section{SESSION 6 DIGITAL HOLOGRAPHY II}

10944 ОT Quasi noise-free reconstruction of long-wavelength digital holograms [10944-28]

10944 OU Kilohertz dynamic Fourier filter for synthetic-aperture binary hologram reconstruction [10944-29]

10944 OV Experimental characterization of leaky-mode spatial light modulators fabricated via direct laser writing [10944-30]

10944 OW Simulating the effects of statistical characteristics of random phases on speckle noise in computer-generated hologram [10944-31]

\section{POSTER SESSION}

10944 OX Holographic camera for non-contact measurement of nanoscale surface heights [10944-32] 
10944 OY Study of holographic gratings of ammonium dichromate varying the hydrolysis concentration of the PVA (poly (vinyl alcohol) [10944-33]

$109440 Z$ Holographic gratings recorded with pineapple juice and artificial dye [10944-34]

$1094410 \quad$ Fourier holograms in grenetina doped with potassium dichromate [10944-35]

$1094411 \quad$ Three-dimensional wavefronts implementation [10944-36]

1094412 3D image encryption based on computer-generated hologram in the fractional Fourier domain [10944-37]

$1094413 \quad$ Near-eye holographic display device by simple optical system using HOE [10944-38]

$1094414 \quad$ Wide axial dynamic range digital holography using multicascade-linked synthetic wavelengths and optical wavelength [10944-39]

$1094415 \quad$ Nopal adhesive with natural pigment films for holographic recording [10944-40]

$1094416 \quad$ Image quality enhancement for digital holographic display using multiple wavefront recording planes method [10944-41] 
Proc. of SPIE Vol. 10944 1094401-6

Downloaded From: https://www.spiedigitallibrary.org/conference-proceedings-of-spie on 25 Apr 2023 Terms of Use: https://www.spiedigitallibrary.org/terms-of-use 


\title{
Authors
}

Numbers in the index correspond to the last two digits of the seven-digit citation identifier (CID) article numbering system used in Proceedings of SPIE. The first five digits reflect the volume number. Base 36 numbering is employed for the last two digits and indicates the order of articles within the volume. Numbers start with 00, 01, 02, 03, 04, 05, 06, 07, 08, 09, OA, OB...0Z, followed by 10-12, 20-2Z, etc.

\author{
Abraham, E., 14 \\ Ahn, Sunggyun, 00 \\ Alim, Marvin D., 05 \\ Ambrosio-González, Mario A., $0 Z$ \\ Amos, Vivian, OD \\ Bablumyan, Arkady, $\mathrm{OH}$ \\ Baselt, T., OU \\ Bianco, Vittorio, OT \\ Bigler, Colton M., $\mathrm{OH}$ \\ Blanche, Pierre-Alexandre, $\mathrm{OH}$ \\ Bolotova, Anastasiia, OG \\ Bougdid, Y., 03 \\ Bove Jr., V. Michael, OV \\ Bruder, Friedrich-Karl, 02 \\ Cao, Liangcai, OA \\ Carson, Jeffrey J. L., OQ, OX \\ Chung, Jae-Seung, OG \\ Coe-Sullivan, Seth, OF \\ Conde-Cuatzo, María G., OY, OZ \\ Dashdavaa, Erkhembaatar, OM, 16 \\ Datta, Bianca, OV \\ Davydova, A. Yu., OL \\ Dimov, Fedor, OF \\ Diop, Mamadou, OQ, OX \\ Draper, Craig, $\mathrm{OH}$ \\ Druzhin, Vladislav, OG \\ Dubynin, Sergey, OG \\ Dyomin, V. V., OL \\ Elgcrona, Gunnar, 08 \\ Ferraro, Pietro, OT \\ Finizio, Andrea, OT \\ Fuentes-Tapia, Israel, 09, 0Y, 0Z \\ Furuta, Kazuya, 13 \\ Gentet, Philippe, OE \\ Gentet, Yves, OE \\ Gerhardt, Nils C., OJ \\ Glugla, David J., 05 \\ Göring, Lena, OJ \\ Hahn, Joonku, 00 \\ Halim, M., 03 \\ Hansen, Sven, 02 \\ Hartmann, P., OU \\ $\mathrm{He}$, Zehao, OA \\ Hens, Korbinian, 08 \\ Hergert, John E., 05 \\ Hofmann, Martin R., 0J \\ Horimai, Hideyoshi, 13 \\ Ibarra-Torres, Juan Carlos, 10 \\ Ibrahim, D. G. A., 14
}

Iwata, T., 14

Jadav, Prashant, OD

Jeon, Seok-Hee, OM

Jeong, Jong-Rea, 16

Jiang, Qiang, OA

Jin, Guofan, OA

Jolly, Sundeep, OV

Joung, Jinbeom, OE

Kabardiadi-Virkovski, A., OU

Kasezawa, Toshihiro, 13

Kawahito, Y., 14

Khuderchuluun, Anar, OM, 16

Kim, Mugeon, 00

Kim, Nam, OM, 12, 16

Kim, Sunil, OG

Kim, Yun-Tae, OG

Kiss, M. Zs., Ol

Kleinschmidt, Tim, 02

Kopenkin, Sergey, OG

Künzel, Roland, 02

Lee, Chang-Kun, $0 \mathrm{G}$

Lee, Hong-Seok, OG

Lee, Seung-Hyun, OE

Lee, Yann, ON

Lewis, Jonathan, 08

Lim, Sungjin, 00

Lim, Young-Tae, OM

Mahfoud, T., 03

Malinina, Polina, $0 G$

Manecke, Christel, 02

Mann, Micah S., $\mathrm{OH}$

Martinez, Christophe, ON

McLeod, Robert R., 05

Mejias-Brisuela, Nildia Yamilet, 09

Memmolo, Pasquale, OT

Meynard, Basile, ON

Minamikawa, T., 14

Minoshima, K., 14

Mitobe, Masaya, OR, OW

Mizuno, T., 14

Mizutani, Y., 14

Montaño-Flores, Beatriz, 10

Moujdi, S., 03

Nelsen, B., OU

Nesterenko, D., 03

Neutsch, Krisztian, OJ

Oe, R., 14

Ogawa, T., 14

Olivares-Pérez, Arturo, 09, OY, 0Z, 10, 11, 15 
Olshukov, A. S., OL

Omidi, Parsa, OQ, OX

Orselli, Enrico, 02

Ortiz-Gutiérrez, Mauricio, 09, 10

Padilla-Velasco, A. L., 15

Padiyar, Joy, OF

Parthiban, Vik, OV

Paturzo, Melania, OT

Pérez-Cortés, Mario, 10

Piao, Mei-Lan, 12

Piao, Yan-Ling, 12, 16

Pioaru, loana, OC

Polovtsev, I. G., OL

Putilin, Andrey, OG

Rahmouni, A., 03

Rewitz, Christian, 02

Richardson, Martin, OD

Ricks, Allen, 08

Rölle, Thomas, 02

Russo, Juan M., OF

Sakamoto, Yuji, OR, OW, 13

Salgado-Verduzco, Marco Antonio, 10

Sanchez, Martin, OF

Sekkat, Z., 03

Seo, Juwon, OG

Seo, Wontaek, $0 G$

Sherliker, Ben, 08

Shibuya, K., 14

Shin, Bongsu, OG

Smalley, Daniel, OV

Sperling, Jaroslaw, 08

Stokes, David Taliesin, 0C

Sullivan, Amy C., 05

Sung, Geeyoung, OG

Tamagawa, Kohei, OW

Taudt, Ch., OU

Toxqui-López, Santa, 09, 0Y, 0Z, 15

Tranelis, Marlon J., OJ

Trovato, C., 14

Villa Hernández, Joan Manuel, 09, 11

Waasem, Niklas, 08

Wang, Hui, OQ, OX

Wu, Hui-Ying, 16

Yamagiwa, M., 14

Yamamito, H., 14

Yasui, T., 14

Zhao, Yu, 16 


\section{Conference Committee}

Symposium Chairs

Connie J. Chang-Hasnain, University of California, Berkeley (United States)

Graham T. Reed, Optoelectronics Research Centre, University of Southampton (United Kingdom)

Symposium Co-chairs

Sailing He, KTH Royal Institute of Technology (Sweden) and Zhejiang University (China)

Yasuhiro Koike, Keio University (Japan)

Program Track Chair

Liang-Chy Chien, Kent State University (United States)

Conference Chairs

Hans I. Bjelkhagen, Glyndwr University (United Kingdom) and Hansholo Consulting Ltd. (United Kingdom)

V. Michael Bove Jr., MIT Media Laboratory (United States)

Conference Program Committee

Maria Isabel Azevedo, Universidade de Aveiro (Portugal)

David Brotherton-Ratcliffe, Geola Technologies Ltd. (United Kingdom)

Frank C. Fan, Dymek Company Ltd. (China)

Gerald L. Heidt, Wasatch Photonics, Inc. (United States)

Toshio Honda, Toppan Printing Company, Ltd. (Japan)

Fujio Iwata, Toppan Printing Company, Ltd. (Japan)

Michael A. Klug, Magic Leap, Inc. (United States)

Alkiviadis Lembessis, The Hellenic Institute of Holography (Greece)

Deanna McMillen, EOTech, Inc. (United States)

Martina L. Mrongovius, RMIT University (Australia) and Center for the Holographic Arts (United States) and Academy of Media Arts, Cologne KHM (Germany)

Hiroshi Yoshikawa, Nihon University (Japan)

Session Chairs

1 Materials and Processes I

Hans I. Bjelkhagen, Glyndwr University (United Kingdom) 
2 Materials and Processes II

Hans I. Bjelkhagen, Glyndwr University (United Kingdom)

3 Art and Perception

Hans I. Bjelkhagen, Glyndwr University (United Kingdom)

4 Applications

V. Michael Bove Jr., MIT Media Laboratory (United States)

5 Digital Holography I

V. Michael Bove Jr., MIT Media Laboratory (United States)

6 Digital Holography II

V. Michael Bove Jr., MIT Media Laboratory (United States) 